\title{
LIGHTing up lipid metabolism
}

Atherosclerosis is characterized by the dysregulation of lipid metabolism (dyslipidaemia), in combination with inflammation. Recent studies have linked members of the tumournecrosis factor (TNF) superfamily to the development of atherosclerosis, but the exact mechanism is unknown. New research, published in Science, now shows that signalling through lymphotoxin- $\beta$ receptor (LT $\beta$ R) by members of the TNF superfamily contributes directly to lipid metabolism.

Lymphotoxin (LT) and LIGHT, which are members of the TNF superfamily and are primarily expressed by $\mathrm{T}$ cells, have both been shown to bind to LT $\beta$ R. Using transgenic mice that overexpress LIGHT in the T-cell lineage, the authors showed that T-cell-derived LIGHT promotes dyslipidaemia. These mice

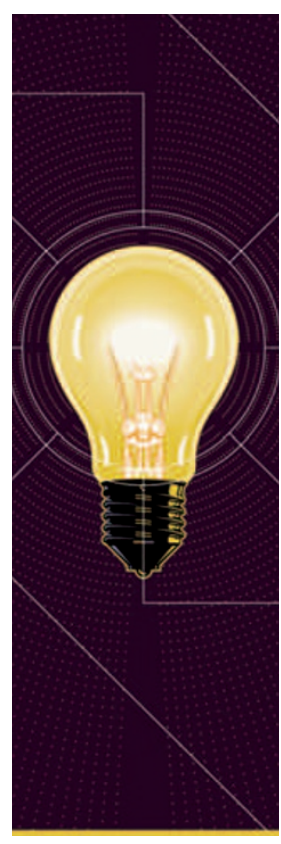

expressed higher levels of cholesterol and triglycerides in the blood. In addition, the transfer of $\mathrm{T}$ cells from LIGHT-transgenic mice to wild-type mice resulted in elevated plasma cholesterol concentrations in the wild-type mice.

To further examine the role of LIGHT in mediating dyslipidaemia, the LIGHT-transgenic mice were crossed with $L t b r^{-1-}$ mice. The level of cholesterol and triglycerides in the blood of these mice was similar to wild-type mice, indicating that LT $\beta \mathrm{R}$ mediated signalling is required for LIGHT-dependent dyslipidaemia.

Because of the association of lipid metabolism with the liver, the authors next examined hepatic gene expression profiles in the LIGHT-transgenic mice. In these mice, the expression level of hepatic lipase, a key enzyme in lipid metabolism, was only 5-10\% of that of wild-type mice. Further studies indicated that the direct interaction of LIGHT on T cells with LT $\beta R$ on hepatocytes inhibited the expression of hepatic lipase.

Administration of an LT $\beta$ Rimmunoglobulin fusion protein, which acts as a soluble decoy protein and blocks both LT- and LIGHTinduced signalling, increased the expression of hepatic lipase and reversed dyslipidaemia in a mouse model of atherosclerosis.

So, the data indicate that $\mathrm{T}$ cells in the liver, through the expression of LT $\beta R$ ligands, have an important role in regulating hepatic-mediated lipoprotein homeostasis, and that agents that block the activation of LT $\beta \mathrm{R}$ might have therapeutic potential in the treatment of dyslipidaemia and atherosclerosis.

Olive Leavy

ORIGINAL RESEARCH PAPER Lo, J. C. et al.

Lymphotoxin $\beta$ receptor-dependent control of lipid homeostasis. Science 316, 285-288 (2007) 